\section{Clinical trials begin in Norway}

A NEW leprosy vaccine developed under the World Health Organization's Programme for Research and Training in Tropical Diseases has started initial trials with human volunteers in Norway. This marks an important step towards the organization's objective of eradicating leprosy, which now afflicts about 12 million people.

The vaccine was developed at the $\mathrm{Na}$ tional Institute for Medical Research in London by a team headed by Dr Richard Rees. Progress towards a vaccine was accelerated in the $1970 \mathrm{~s}$ with the discovery that the causal agent, Mycobacterium leprae - which cannot be grown in vitro could be cultured in armadillos. $M$. leprae grows best at a temperature below that of most mammals, which may explain its preference for the body extremities in humans. Armadillos have a body temperature sufficiently low for $M$. leprae to grow in the liver and spleen.

The new vaccine is based on killed $M$. leprae extracted from armadillos. The crucial step in the development programme - which has taken five years - came with the perfection of a method of inactivating enzymes from the tissues of the host armadillo that previously damaged the bacterial antigens when samples of infected tissue were homogenized. The $M$. leprae are killed by radiation and autoclaved. Tests with laboratory animals show that, surprisingly, the killed bacteria are more immunogenic than live $M$. leprae.

The vaccine is now being manufactured by the Wellcome Foundation Ltd in England, from armadillos kept at the Centre for Applied Microbiology and Research at Porton Down. Clinical trials are expected to start in Britain and the United States before the end of the year. These countries were selected because they have very different BCG tuberculosis immunization programmes, and $M$. leprae is known to interact in some way with BCG. Although the vaccine is intended primarily for use in developing countries, initial trials in developed countries should throw light on safety and on the immune response of the system.

The need for a new weapon against leprosy has been made more urgent as $M$. leprae has evolved resistance to the drug most widely used to treat the disease, dapsone. Other approaches are also being pursued: Dr $\mathrm{R}$ Curtiss at Alabama State University is using genetic engineering techniques to produce $M$. leprae antigens. This work is also financed by the World Health Organization.

Dr J Convit, at the Pan-American Centre for Research and Training in Leprosy and Tropical diseases in Caracas, Venezuela, has been using a vaccine similar to that developed in Britain in combination with live $\mathrm{BCG}$ tuberculosis vaccine for secondary prevention of lepromatous $0028-0836 / 83 / 140469-02 \$ 01.00$ leprosy in patients already affected by the disease. Patients with this form of leprosy suffer from a suppression of the immune system, and the $\mathrm{BCG} / M$. leprae combination appears to restore immune defences. Early results are said to be promising.

Tim Beardsley

\section{Nucleotide sequences

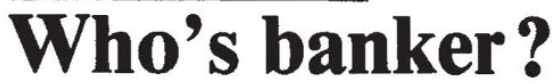

\section{Washington}

WHEN the National Institutes of Health (NIH) last July awarded a five-year contract worth $\$ 3.14$ million to Bolt, Beranek and Newman (BB\&N), a private company, to set up a database of nucleic acid sequences, the promise that researchers in genetics would have a central library of sequences seemed to take a major step forward. But now the contract has been challenged by another applicant.

In approving BB\&N's proposal, NIH turned down a rival proposal from an existing repository of nucleic acid sequences, the National Biomedical Research Foundation (NBRF), located at Georgetown University here. This is an old and well respected library of both nucleic acid and protein sequences and is contesting NIH's award to BB\&N. It will be up to the Comptroller General to decide if NIH followed correct procedures in making their decision.

Meanwhile, NBRF is in trouble. Last March, its founder and principal
THE European Space Agency (ESA) has selected ISO, an expensive infrared astronomical observatory satellite, for its next scientific mission, despite protests that ESA is neglecting planetary science (see Nature 24 February, p.647).

ESA's list of scientific satellites now comprises: Exosat, an $\mathbf{X}$-ray satellite for launch by the US National Aeronautics and Space Administration (NASA) at the end of May this year; Giotto, to fly past Halley's Comet, for launch mid-1985; Hipparcos, for astrometry, for launch towards the end of 1986; and now ISO, for the "early 1990s". ESA is also participating in the NASA Space Telescope. The four other proposals before the ESA Science Programme Committee last week, Disco (for solar oscillations), Magellan (UV astronomy), X-80 (X-ray astronomy) and Kepler (a Mars lander), now seem doomed, as another ESA scientific mission will not be selected for another two or three years with no prospect of a launch before 1995 . The long gaps between Hipparcos, ISO and the following project are determined partly by the cost of ISO: some 250 million ac-

investigator, Dr Margaret Dayhoff, died at the age of 57. Dayhoff had started the protein data collection in the early 1960 s and in 1965 published the first edition of the Atlas of Protein Sequences. A new volume is to be published soon. NIH supported Dayhoff's protein data bank for many years, and still do, but at a low level. And the fate of the databank of nucleic acid sequences is now unclear. Project director Winona Barker says that it is still being updated and has entries of better quality than the BB\&N databank. In December it included 1.2 million nucleotides under 862 entries, all annotated as to where the interesting sites are and with other comments. The data are available for a fee.

Meanwhile, the BB\&N databank is growing like Topsy. BB\&N subcontracted some of the work to the Los Alamos National Laboratory. Los Alamos project director Walter Goad says that it now has 1.37 million bases of which 90 per cent are annotated. More than a hundred subscribers use it through NIH's computer at BB\&N's offices in Cambridge, Massachusetts.

The databank incorporates information from the only other collection of comparable size, that at the European Molecular Biology Organization in Heidelberg, West Germany. Both Intelligenetics Inc., a Palo Alto company servicing biotechnology companies, and another data effort at the University of Wisconsin use the Los Alamos data. But if Dayhoff's successors succeed in having the NIH award overturned, this developing pattern will itself be shaken up.

Deborah Shapley

\title{
ESA settles for astrophysics
}

counting units (AU) ( $£ 137.5$ million), compared with ESA's science budget of under 100 million AU. Kepler would have cost only 150 million $A U$.

ISO will be comparable with the currently operating IRAS inf rared satellite (a joint UK-Netherlands-US collaboration): it will be cooled with liquid hydrogen and helium to $13 \mathrm{~K}$, and have a lifetime determined by the evaporation rate of the coolants. It will be 100 times more sensitive than IRAS and so able to study extragalactic objects - thus complementing the Space Telescope. Judging from the February report of ESA's Astronomy Working Group, one aim is to give Europe a commanding position in infrared astronomy.

Meanwhile, European planetary science may "wither away", says Professor Fred Taylor, acting head of the department of atmospheric physics at the University of Oxford and supporter of Kepler. The only remaining planetary mission in view is NASA's Galileo, due to orbit Jupiter in 1989. The only real solution is to increase ESA's mandatory science budget, he says. Robert Walgate 